\title{
Intuitionistic fuzzy implications revisited. Part 1
}

\author{
Krassimir Atanassov ${ }^{1}$, Eulalia Szmidt ${ }^{2}$, Janusz Kacprzyk ${ }^{2}$ \\ and Nora Angelova ${ }^{3}$ \\ ${ }^{1}$ Department of Bioinformatics and Mathematical Modelling \\ Institute of Biophysics and Biomedical Engineering \\ Bulgarian Academy of Sciences \\ Acad. G. Bonchev Str., B1. 105, Sofia-1113, Bulgaria \\ e-mail: krat@bas.bg \\ ${ }^{2}$ Systems Research Institute, Polish Academy of Sciences \\ ul. Newelska 6, 01-447 Warsaw, Poland \\ and \\ Warsaw School of Information Technology \\ ul. Newelska 6, 01-447 Warsaw, Poland \\ e-mails: \{szmidt, kacprzyk\} @ibspan.waw.pl \\ ${ }^{3}$ Faculty of Mathematics and Informatics, Sofia University \\ 5 James Bourchier Blvd., Sofia 1164, Bulgaria \\ e-mail: nora.angelova@fmi.uni-sofia.bg
}

Received: 6 March 2019 Accepted: 10 April 2019

Abstract: Definitions of two intuitionistic fuzzy implications, published during the last 15 years, are revisited. It is checked which existing by the moment intuitionistic fuzzy implications (190 in number) satisfy a well-known tautology in first order logic.

Keywords: Intuitionistic fuzzy implication, Intuitionistic fuzzy logic.

2010 Mathematics Subject Classification: 03E72.

\section{Introduction}

In [3], 185 different intuitionisic fuzzy implications are given. After its publishing, five new implications are introduced in [2-8]. In [3], the problem for correctness of these implications is 
discussed. Some criteria are shown as suitable for this problem.

The pair $A=\langle a, b\rangle$ is called an intuitionisic fuzzy pair, if $a, b, a+b \in[0,1]$. It is:

a tautology if and only if (iff) $a=1$ and $b=0$, and

an intuitionistic fuzzy tautology (IFT) iff $a \geq b$.

For the implications, for example, it is obligatory that they satisfy the following condition

$$
\begin{aligned}
& \langle 0,1\rangle \rightarrow\langle 0,1\rangle=\langle 1,0\rangle, \\
& \langle 0,1\rangle \rightarrow\langle 1,0\rangle=\langle 1,0\rangle, \\
& \langle 1,0\rangle \rightarrow\langle 0,1\rangle=\langle 0,1\rangle, \\
& \langle 1,0\rangle \rightarrow\langle 1,0\rangle=\langle 1,0\rangle,
\end{aligned}
$$

i.e., they behave as standard tautologies.

In the present research, we will formulate new condition for correctness of the intuitionisic fuzzy implications.

\section{Main results}

Let $A=\langle a, b\rangle$, and $B=\langle c, d\rangle$ where $a, b, c, d, a+b, c+d \in[0,1]$, be intuitionisic fuzzy pairs. Initially, following [12], we must mention that two of implications from [3] coincide in spite of the fact that they have different records there. They are

$$
\langle a, b\rangle \rightarrow_{40}\langle c, d\rangle=\langle 1-\operatorname{sg}(a+d-1), 1-\overline{\operatorname{sg}}(a+d-1)\rangle
$$

and

$$
\langle a, b\rangle \rightarrow_{173}\langle c, d\rangle=\langle\overline{\operatorname{sg}}(a+d-1), \operatorname{sg}(a+d-1)\rangle,
$$

where

$$
\begin{aligned}
& \operatorname{sg}(x)= \begin{cases}1 & \text { if } x>0 \\
0 & \text { if } x \leq 0\end{cases} \\
& \overline{\operatorname{sg}}(x)= \begin{cases}0 & \text { if } x>0 \\
1 & \text { if } x \leq 0\end{cases}
\end{aligned}
$$

Really, for each variable $x$ :

$$
1-\operatorname{sg}(x)=\overline{\operatorname{sg}}(x)
$$

In the first part of our research, we will check which implications from [3] satisfy the wellknown formula

$$
((\neg \neg A \rightarrow A) \rightarrow(\neg \neg A \vee \neg A)) \rightarrow(\neg \neg A \vee \neg A)
$$

that in first order logic (see, e.g., [11]) is a tautology.

Theorem 1. Implication $\rightarrow_{i}$ satisfy (1) for $i=2,3,8,11,14,15,16,19,20,23,31,32,34,37$, 40, $41,42,43,44,45,47,48,52,55,56,57,62,63,65,68,69,74,77,83,84,88,90,97,99,171,172$, $174,175,176,177,178,179,180,181,182,183,185$ as a standard tautology. 
Proof. We will check Theorem 1 for $i=2$. The remaining checks are analogous. The implication $\rightarrow_{2}$ and the negation $\neg_{2}$, generated by it, have the forms (see [3]):

$$
A \rightarrow_{2} B=\langle\overline{\operatorname{sg}}(a-c), d \operatorname{sg}(a-c)\rangle
$$

where $B=\langle c, d\rangle$ and

$$
\neg_{2} A=\langle\overline{\operatorname{sg}}(a), \operatorname{sg}(a)\rangle .
$$

Let

$$
\begin{gathered}
Z \equiv\left(\left(\neg_{2} \neg_{2} A \rightarrow_{2} A\right) \rightarrow_{2}\left(\neg_{2} \neg_{2} A \vee \neg_{2} A\right)\right) \rightarrow_{2}\left(\neg_{2} \neg_{2} A \vee \neg_{2} A\right) \\
=\left(\left(\neg_{2} \neg_{2}\langle a, b\rangle \rightarrow_{2}\langle a, b\rangle\right) \rightarrow_{2}\left(\neg_{2} \neg_{2}\langle a, b\rangle \vee \neg_{2}\langle a, b\rangle\right)\right) \rightarrow_{2}\left(\neg_{2} \neg_{2}\langle a, b\rangle \vee \neg_{2}\langle a, b\rangle\right) \\
=\left(\left(\neg_{2}\langle\overline{\operatorname{sg}}(a), \operatorname{sg}(a)\rangle \rightarrow_{2}\langle a, b\rangle\right) \rightarrow_{2}\left(\neg_{2}\langle\overline{\operatorname{sg}}(a), \operatorname{sg}(a)\rangle \vee\langle\overline{\operatorname{sg}}(a), \operatorname{sg}(a)\rangle\right)\right) \\
\rightarrow_{2}\left(\neg_{2}\langle\overline{\operatorname{sg}}(a), \operatorname{sg}(a)\rangle \vee\langle\overline{\operatorname{sg}}(a), \operatorname{sg}(a)\rangle\right) \\
=\left(\left(\langle\overline{\operatorname{sg}}(\overline{\operatorname{sg}}(a)), \operatorname{sg}(\overline{\operatorname{sg}}(a))\rangle \rightarrow_{2}\langle a, b\rangle\right) \rightarrow_{2}(\langle\overline{\operatorname{sg}}(\overline{\operatorname{sg}}(a)), \operatorname{sg}(\overline{\operatorname{sg}}(a))\rangle \vee\langle\overline{\operatorname{sg}}(a), \operatorname{sg}(a)\rangle)\right) \\
\rightarrow_{2}(\langle\overline{\operatorname{sg}}(\overline{\operatorname{sg}}(a)), \operatorname{sg}(\overline{\operatorname{sg}}(a))\rangle \vee\langle\overline{\operatorname{sg}}(a), \operatorname{sg}(a)\rangle) \\
=\left(\left(\langle\operatorname{sg}(a), 1-\operatorname{sg}(a)\rangle \rightarrow_{2}\langle a, b\rangle\right) \rightarrow_{2}(\langle\operatorname{sg}(a), 1-\operatorname{sg}(a)\rangle \vee\langle\overline{\operatorname{sg}}(a), \operatorname{sg}(a)\rangle)\right) \\
\quad \rightarrow_{2}(\langle\operatorname{sg}(a), 1-\operatorname{sg}(a)\rangle \vee\langle\overline{\operatorname{sg}}(a), \operatorname{sg}(a)\rangle) \\
=\left(\langle\overline{\operatorname{sg}}(\operatorname{sg}(a)-a), b \operatorname{sg}(\operatorname{sg}(a)-a)\rangle \rightarrow_{2}\langle\max (\operatorname{sg}(a), \overline{\operatorname{sg}}(a)), \min (1-\operatorname{sg}(a), \operatorname{sg}(a))\rangle\right) \\
\rightarrow_{2}\langle\max (\operatorname{sg}(a), \overline{\operatorname{sg}}(a)), \min (1-\operatorname{sg}(a), \operatorname{sg}(a))\rangle \\
=\left(\langle\overline{\operatorname{sg}}(\operatorname{sg}(a)-a), b \operatorname{sg}(\operatorname{sg}(a)-a)\rangle \rightarrow_{2}\langle\max (\operatorname{sg}(a), \overline{\operatorname{sg}}(a)), \min (1-\operatorname{sg}(a), \operatorname{sg}(a))\rangle\right) \\
\rightarrow_{2}\langle\max (\operatorname{sg}(a), \overline{\operatorname{sg}}(a)), \min (1-\operatorname{sg}(a), \operatorname{sg}(a))\rangle . \\
\text { If } a=0, \operatorname{then} \operatorname{sg}(a)=0, \overline{\operatorname{sg}}(a)=1 \text { and }
\end{gathered}
$$

$$
\begin{gathered}
Z=(\langle\overline{\operatorname{sg}}(0), b \operatorname{sg}(0)\rangle \rightarrow\langle\max (0,1), \min (1,0)\rangle) \rightarrow\langle\max (0,1), \min (1,0)\rangle \\
=(\langle 1,0\rangle \rightarrow\langle 1,0\rangle) \rightarrow\langle 1,0\rangle \\
=\langle\overline{\operatorname{sg}}(1-1), 0 \cdot \operatorname{sg}(1-1)\rangle \rightarrow\langle 1,0\rangle \\
=\langle 1,0\rangle \rightarrow\langle 1,0\rangle \\
=\langle\overline{\operatorname{sg}}(1-1), 0 \cdot \operatorname{sg}(1-1)\rangle=\langle 1,0\rangle .
\end{gathered}
$$

Therefore, $Z$ is a tautology.

Now, we will give an example in which, e.g., implication

$$
A \rightarrow_{12} B=\langle\max (b, c), 1-\max (b, c)\rangle
$$

and the negation

$$
\neg_{10} A=\langle b, 1-b\rangle
$$

generated by it, do not satisfy (1). 
Let

$$
\begin{gathered}
Z \equiv\left(\left(\neg_{4} \neg_{4} A \rightarrow_{12} A\right) \rightarrow_{12}\left(\neg_{4} \neg_{4} A \vee \neg_{4} A\right)\right) \rightarrow_{12}\left(\neg_{4} \neg_{4} A \vee \neg_{12} A\right) \\
=\left(\left(\neg_{4} \neg_{4}\langle a, b\rangle \rightarrow_{12}\langle a, b\rangle\right) \rightarrow_{12}\left(\neg_{4} \neg_{4}\langle a, b\rangle \vee \neg_{4}\langle a, b\rangle\right)\right) \\
\rightarrow_{12}\left(\neg_{4} \neg_{4}\langle a, b\rangle \vee \neg_{4}\langle a, b\rangle\right) \\
=\left(\left(\neg_{4}\langle b, 1-b\rangle \rightarrow_{12}\langle a, b\rangle\right) \rightarrow_{12}\left(\neg_{4}\langle b, 1-b\rangle \vee\langle b, 1-b\rangle\right)\right) \rightarrow_{12}\left(\neg_{4}\langle b, 1-b\rangle \vee\langle b, 1-b\rangle\right) \\
=\left(\left(\langle 1-b, b\rangle \rightarrow_{12}\langle a, b\rangle\right) \rightarrow_{12}(\langle 1-b, b\rangle \vee\langle b, 1-b\rangle)\right) \rightarrow_{12}(\langle 1-b, b\rangle \vee\langle b, 1-b\rangle) \\
=\left(\langle\max (a, b), 1-\max (a, b)\rangle \rightarrow_{12}\langle\max (b, 1-b), \min (b, 1-b)\rangle\right) \\
\rightarrow \rightarrow_{12}\langle\max (b, 1-b), \min (b, 1-b)\rangle \\
=(\langle\max (1-\max (a, b), \max (b, 1-b)), 1-\max (1-\max (a, b), \max (b, 1-b))\rangle \\
\rightarrow_{12}\langle\max (b, 1-b), \min (b, 1-b)\rangle \\
=(\langle\max (\min (1-a, 1-b), \max (b, 1-b)), 1-\max (\min (1-a, 1-b), \max (b, 1-b))\rangle \\
\rightarrow_{12}\langle\max (b, 1-b), \min (b, 1-b)\rangle
\end{gathered}
$$

(because, for $a, b, a+b \in[0,1]: \min (1-a, 1-b) \leq 1-b \leq \max (b, 1-b)$ )

$$
\begin{gathered}
=\langle\max (b, 1-b), 1-\max (b, 1-b)\rangle \rightarrow_{12}\langle\max (b, 1-b), \min (b, 1-b)\rangle \\
=\langle\max (1-\max (b, 1-b), \max (b, 1-b)), 1-\max (1-\max (b, 1-b), \max (b, 1-b))\rangle \\
=\langle\max (\min (b, 1-b), \max (b, 1-b)), 1-\max (\min (b, 1-b), \max (b, 1-b))\rangle \\
=\langle\max (b, 1-b), 1-\max (b, 1-b)\rangle \\
=\langle\max (b, 1-b), \min (b, 1-b)\rangle \neq\langle 1,0\rangle
\end{gathered}
$$

for $0 \leq b<1$, i.e., (1) is not a tautology.

Theorem 2. Implication $\rightarrow_{i}$ satisfies (1) for $i=1,2,3,4,5,6,7,8,9,11,12,13,14,15,16,17,18$, $19,20,21,22,23,24,25,26,27,28,29,30,31,32,33,34,35,36,37,38,40,41,42,43,44,45,46$, $47,48,49,50,51,52,53,55,56,57,61,62,63,64,65,66,67,68,69,71,72,74,75,76,77,79,80$, $81,82,83,84,85,86,88,89,90,91,94,95,97,98,99,100,101,102,103,104,105,106,107,109$, $110,111,112,113,114,115,116,117,118,119,120, \quad 121,122,123,124,125,126,127,128,129$, $130,131,132,133,134,135,136,137, \quad 141,146,147,148,151,158,159,160,161,166,167,168$, $169,170,171,172,174,175,176,177,178,179,180,181,182,183,184,185$ as IFT.

Proof. We will check Theorem 2 for $i=1$. The remining checks are analogous. The implication $\rightarrow_{1}$ and the negation $\neg_{1}$ generated by it, have the forms (see [3]):

$$
A \rightarrow_{1} B=\langle\max (b, \min (a, c)), \min (a, d)\rangle,
$$

where $B$ is defined above and

$$
\neg_{1} A=\langle b, a\rangle .
$$


Let

$$
\begin{aligned}
& Z \equiv\left(\left(\neg_{1} \neg_{1} A \rightarrow_{1} A\right) \rightarrow_{1}\left(\neg_{1} \neg_{1} A \vee \neg_{1} A\right)\right) \rightarrow_{1}\left(\neg_{1} \neg_{1} A \vee \neg_{1} A\right) \\
& =\left(\left(\neg_{1} \neg_{1}\langle a, b\rangle \rightarrow_{1}\langle a, b\rangle\right) \rightarrow_{1}\left(\neg_{1} \neg_{1}\langle a, b\rangle \vee \neg_{1}\langle a, b\rangle\right)\right) \\
& \rightarrow_{1}\left(\neg_{1} \neg_{1}\langle a, b\rangle \vee \neg_{1}\langle a, b\rangle\right) \\
& =\left(\left(\neg_{1}\langle b, a\rangle \rightarrow_{1}\langle a, b\rangle\right) \rightarrow_{1}\left(\neg_{1}\langle b, a\rangle \vee\langle b, a\rangle\right)\right) \\
& \rightarrow_{1}\left(\neg_{1}\langle b, a\rangle \vee\langle b, a\rangle\right) \\
& =\left(\left(\langle a, b\rangle \rightarrow_{1}\langle a, b\rangle\right) \rightarrow_{1}(\langle a, b\rangle \vee\langle b, a\rangle)\right) \\
& \rightarrow_{1}(\langle a, b\rangle \vee\langle b, a\rangle) \\
& =\left(\langle\max (b, \min (a, a)), \min (a, b)\rangle \rightarrow_{1}\langle\max (a, b), \min (a, b)\rangle\right) \rightarrow_{1}\langle\max (a, b), \min (a, b)\rangle \\
& =\left(\langle\max (a, b), \min (a, b)\rangle \rightarrow_{1}\langle\max (a, b), \min (a, b)\rangle\right) \rightarrow_{1}\langle\max (a, b), \min (a, b)\rangle \\
& =(\langle\max (\min (a, b), \min (\max (a, b), \max (a, b))), \min (\max (a, b), \min (a, b))\rangle) \\
& \rightarrow_{1}\langle\max (a, b), \min (a, b)\rangle \\
& =\langle\max (\min (\max (a, b), \min (a, b)), \\
& \min (\max (\min (a, b), \min (\max (a, b), \max (a, b))), \max (a, b))), \\
& \min (\max (\min (a, b), \min (\max (a, b), \max (a, b))), \min (a, b))\rangle \\
& =\langle\max (\min (a, b), \min (\max (\min (a, b), \max (a, b)), \max (a, b))), \\
& \min (\max (\min (a, b), \max (a, b)), \min (a, b))\rangle \\
& =\langle\max (\min (a, b), \min (\max (a, b), \max (a, b))), \\
& \min (\max (\min (a, b), \max (a, b)), \min (a, b))\rangle \\
& =\langle\max (\min (a, b), \max (a, b)), \min (\max (a, b), \min (a, b))\rangle \\
& =\langle\max (a, b), \min (a, b)\rangle .
\end{aligned}
$$

Therefore, $Z$ is an IFT.

Now, we will give an example in which, e.g., the implication

$$
A \rightarrow_{60} B=\langle\max (\overline{\operatorname{sg}}(1-b), \overline{\operatorname{sg}}(1-c)), \min (1-b, \overline{\operatorname{sg}}(c))\rangle
$$

and the negation, generated by it

$$
\neg_{10} A=\langle\overline{\operatorname{sg}}(1-b), 1-b\rangle
$$

(see [3]) do not satisfy (1).

$$
\begin{gathered}
Z \equiv\left(\left(\neg_{10} \neg_{10} A \rightarrow_{60} A\right) \rightarrow_{60}\left(\neg_{10} \neg_{10} A \vee \neg_{10} A\right)\right) \rightarrow_{60}\left(\neg_{10} \neg_{10} A \vee \neg_{10} A\right) \\
\left.=\left(\left(\neg_{10}\right\urcorner_{10}\langle a, b\rangle \rightarrow_{60}\langle a, b\rangle\right) \rightarrow_{60}\left(\neg_{10} \neg_{10}\langle a, b\rangle \vee \neg_{10}\langle a, b\rangle\right)\right) \\
\rightarrow_{60}\left(\neg_{10} \neg 10\langle a, b\rangle \vee \neg_{10}\langle a, b\rangle\right)
\end{gathered}
$$




$$
\begin{gathered}
=\left(\left(\neg_{10}\langle\overline{\operatorname{sg}}(1-b), 1-b\rangle \rightarrow_{60}\langle a, b\rangle\right) \rightarrow_{60}\left(\neg_{10}\langle\overline{\operatorname{sg}}(1-b), 1-b\rangle \vee\langle\overline{\operatorname{sg}}(1-b), 1-b\rangle\right)\right) \\
\rightarrow_{60}\left(\neg_{10}\langle\overline{\operatorname{sg}}(1-b), 1-b\rangle \vee\langle\overline{\operatorname{sg}}(1-b), 1-b\rangle\right) \\
=\left(\left(\neg_{10}\langle\overline{\operatorname{sg}}(1-b), 1-b\rangle \rightarrow_{60}\langle a, b\rangle\right) \rightarrow_{60}\left(\neg_{10}\langle\overline{\operatorname{sg}}(1-b), 1-b\rangle \vee\langle\overline{\operatorname{sg}}(1-b), 1-b\rangle\right)\right) \\
\rightarrow_{60}\left(\neg_{10}\langle\overline{\operatorname{sg}}(1-b), 1-b\rangle \vee\langle\overline{\operatorname{sg}}(1-b), 1-b\rangle\right) \\
=\left(\left(\langle\overline{\operatorname{sg}}(b), b\rangle \rightarrow_{60}\langle a, b\rangle\right) \rightarrow_{60}(\langle\overline{\operatorname{sg}}(b), b\rangle \vee\langle\overline{\operatorname{sg}}(1-b), 1-b\rangle)\right) \\
\quad \rightarrow_{60}(\langle\overline{\operatorname{sg}}(b), b\rangle \vee\langle\overline{\operatorname{sg}}(1-b), 1-b\rangle) \\
=\left(\langle\max (\overline{\operatorname{sg}}(1-b), \overline{\operatorname{sg}}(1-a)), \min (1-b, \overline{\operatorname{sg}}(a))\rangle \rightarrow_{60}\langle\max (\overline{\operatorname{sg}}(b), \overline{\operatorname{sg}}(1-b)), \min (b, 1-b)\rangle\right) \\
\rightarrow{ }_{60}(\langle\max (\overline{\operatorname{sg}}(b), \overline{\operatorname{sg}}(1-b)), \min (b, 1-b)\rangle \\
=\langle\max (\overline{\operatorname{sg}}(1-\min (1-b, \overline{\operatorname{sg}}(a))), \overline{\operatorname{sg}}(1-\max (\overline{\operatorname{sg}}(b), \overline{\operatorname{sg}}(1-b)))), \\
\min (1-\min (1-b, \overline{\operatorname{sg}}(a)), \overline{\operatorname{sg}}(\max (\overline{\operatorname{sg}}(b), \overline{\operatorname{sg}}(1-b))))\rangle) \\
\quad \rightarrow_{60}\langle\max (\overline{\operatorname{sg}}(b), \overline{\operatorname{sg}}(1-b)), \min (b, 1-b)\rangle \\
=\langle\max (\overline{\operatorname{sg}}(1-\min (1-\min (1-b, \overline{\operatorname{sg}}(a)), \overline{\operatorname{sg}}(\max (\overline{\operatorname{sg}}(b), \overline{\operatorname{sg}}(1-b)))), \\
\overline{\operatorname{sg}}(1-\langle\max (\overline{\operatorname{sg}}(b), \overline{\operatorname{sg}}(1-b)))), \\
\min (1-\min (1-\min (1-b, \overline{\operatorname{sg}}(a)), \overline{\operatorname{sg}}(\max (\overline{\operatorname{sg}}(b), \overline{\operatorname{sg}}(1-b)))), \overline{\operatorname{sg}}(\max (\overline{\operatorname{sg}}(b), \overline{\operatorname{sg}}(1-b)))\rangle .
\end{gathered}
$$

Now, we check the value of

$$
\begin{gathered}
Y_{1} \equiv \max (\overline{\mathrm{sg}}(1-\min (1-\min (1-b, \overline{\mathrm{sg}}(a)), \overline{\operatorname{sg}}(\max (\overline{\mathrm{sg}}(b), \overline{\operatorname{sg}}(1-b))))), \\
\overline{\mathrm{sg}}(1-\max (\overline{\mathrm{sg}}(b), \overline{\mathrm{sg}}(1-b)))) .
\end{gathered}
$$

If $b=0$, then

$$
\begin{gathered}
Y_{1}=\max (\overline{\operatorname{sg}}(1-\min (1-\min (1, \overline{\operatorname{sg}}(a)), \overline{\operatorname{sg}}(\max (1,0)))), \overline{\operatorname{sg}}(1-\max (1,0))) \\
=\max (\overline{\operatorname{sg}}(1-\min (1-\overline{\operatorname{sg}}(a), \overline{\operatorname{sg}}(1))), \overline{\operatorname{sg}}(1-1)) \\
=\max (\overline{\operatorname{sg}}(1-\min (1-\overline{\operatorname{sg}}(a), 0)), 1) \\
=\max (\overline{\operatorname{sg}}(1-0), 1)=1 .
\end{gathered}
$$

If $b=1$, then

$$
\begin{gathered}
Y_{1}=\max (\overline{\operatorname{sg}}(1-\min (1-\min (0, \overline{\operatorname{sg}}(a)), \overline{\operatorname{sg}}(\max (\overline{\operatorname{sg}}(1), \overline{\operatorname{sg}}(0)))), \overline{\operatorname{sg}}(1-\max (\overline{\operatorname{sg}}(1), \overline{\operatorname{sg}}(0)))) \\
=\max (\overline{\operatorname{sg}}(1-\min (1-\min (0, \overline{\operatorname{sg}}(a)), \overline{\operatorname{sg}}(\max (0,1)))), \overline{\operatorname{sg}}(1-\max (0,1))) \\
=\max (\overline{\operatorname{sg}}(1-\min (1-\min (0, \overline{\operatorname{sg}}(a)), \overline{\operatorname{sg}}(1))), \overline{\operatorname{sg}}(1-1)) \\
=\max (\overline{\operatorname{sg}}(1-\min (1-0,0)), 1) \\
=\max (\overline{\operatorname{sg}}(1), 1)=1 .
\end{gathered}
$$


If $0<b<1$, then

$$
\begin{gathered}
Y_{1}=\max (\overline{\operatorname{sg}}(1-\min (1-\min (1-b, \overline{\operatorname{sg}}(a)), \overline{\operatorname{sg}}(\max (0,0)))), \overline{\operatorname{sg}}(1-\max (0,0))) \\
Y_{1}=\max (\overline{\operatorname{sg}}(1-\min (1-\min (1-b, \overline{\operatorname{sg}}(a)), \overline{\operatorname{sg}}(0))), \overline{\operatorname{sg}}(1)) \\
Y_{1}=\max (\overline{\operatorname{sg}}(1-\min (1-\min (1-b, \overline{\operatorname{sg}}(a)), 1)), 0) \\
Y_{1}=\max (\overline{\operatorname{sg}}(1-(1-\min (1-b, \overline{\operatorname{sg}}(a)))), 0) \\
Y_{1}=\overline{\operatorname{sg}}(\min (1-b, \overline{\operatorname{sg}}(a))) .
\end{gathered}
$$

Let

$Y_{2} \equiv \min (1-\min (1-\min (1-b, \overline{\mathrm{sg}}(a)), \overline{\mathrm{sg}}(\max (\overline{\mathrm{sg}}(b), \overline{\operatorname{sg}}(1-b)))), \overline{\mathrm{sg}}(\max (\overline{\mathrm{sg}}(b), \overline{\mathrm{sg}}(1-b))))$.

For $b=0,1$ we can see immediately that $Y_{2}=0$.

If $0<b<1$, then

$$
\begin{gathered}
Y_{2}=\min (1-\min (1-\min (1-b, \overline{\operatorname{sg}}(a)), \overline{\operatorname{sg}}(\max (0,0))), \overline{\operatorname{sg}}(\max (0,0))) \\
=\min (1-\min (1-\min (1-b, \overline{\operatorname{sg}}(a)), \overline{\operatorname{sg}}(\max (0,0))), \overline{\operatorname{sg}}(\max (0,0))) \\
=\min (1-\min (1-\min (1-b, \overline{\operatorname{sg}}(a)), \overline{\operatorname{sg}}(0)), \overline{\operatorname{sg}}(0)) \\
=\min (1-\min (1-\min (1-b, \overline{\operatorname{sg}}(a)), 1), 1) \\
=\min (1-(1-\min (1-b, \overline{\operatorname{sg}}(a))), 1) \\
=\min (1-b, \overline{\operatorname{sg}}(a)) .
\end{gathered}
$$

Therefore,

$$
Z=\langle\overline{\operatorname{sg}}(\min (1-b, \overline{\operatorname{sg}}(a))), \min (1-b, \overline{\operatorname{sg}}(a))\rangle .
$$

If $a=0$, then

$$
\begin{gathered}
Z=\langle\overline{\operatorname{sg}}(\min (1-b, 1)), \min (1-b, 1)\rangle \\
=\langle\overline{\operatorname{sg}}(1-b), 1-b\rangle \\
=\langle 0,1-b\rangle
\end{gathered}
$$

that for $0<b<1$ is not an IFT. So, we have shown that Theorem 2 is not valid for $\rightarrow_{60}$.

\section{Conclusion}

In the second part of the present research, we will determine which intuitionistic fuzzy implications satisfy two other tautologies in first order logic.

Open Problem: In [2] six Cartesian products are defined (the last of them was published in [1]). For which indices $i$ and $j$ the $i$-th implication and $j$-th Cartesian product satisfies equality

$$
\left(A \rightarrow_{i} B\right) \times_{j} C=\left(A \times_{j} C\right) \rightarrow_{i}\left(B \times_{j} C\right)
$$

for every three IFSs $A, B, C$ ? 


\section{Acknowledgements}

This work has been partially supported by the Bulgarian National Science Fund under Grant Ref. No. DN-02-10/2016 "New Instruments for Knowledge Discovery from Data, and their Modelling"

\section{References}

[1] Andonov, V. (2008). On some properties of one Cartesian product over intuitionistic fuzzy sets. Notes on Intuitionistic Fuzzy Sets, 14 (1), 12-19.

[2] Atanassov, K. (2012). On Intuitionistic Fuzzy Sets Theory, Springer, Berlin.

[3] Atanassov, K. (2017). Intuitionistic Fuzzy Logics. Springer, Cham.

[4] Atanassov, K., Angelova, N., Szmidt, E., \& Kacprzyk, J. (2016). Properties of the intuitionistic fuzzy implication $\rightarrow_{186}$. Notes on Intuitionistic Fuzzy Sets, 22 (4), 6-12.

[5] Atanassov, K., Ribagin, S., Doukovska, L., \& Atanassova, V. (2017). Intuitionistic fuzzy implication $\rightarrow_{190}$. Notes on Intuitionistic Fuzzy Sets, 23 (4), 79-83.

[6] Atanassov, K., Szmidt, E., \& Kacprzyk, J. (2016). New Fodor's type of intuitionistic fuzzy implication and negation. Notes on Intuitionistic Fuzzy Sets, 22 (3), 1-8.

[7] Atanassov, K., Szmidt, E., \& Kacprzyk, J. (2017). Intuitionistic fuzzy implication $\rightarrow_{188}$. Notes on Intuitionistic Fuzzy Sets, 23 (1), 6-13.

[8] Atanassov, K., Szmidt, E., \& Kacprzyk, J. (2017). Intuitionistic fuzzy implication $\rightarrow_{187}$. Notes on Intuitionistic Fuzzy Sets, 23 (2), 37-43.

[9] Atanassov, K., Szmidt, E., \& Angelova, N. (2017). Properties of the intuitionistic fuzzy implication $\rightarrow{ }_{187}$. Notes on Intuitionistic Fuzzy Sets, 23 (3), 3-8.

[10] Atanassova, L. (2017). Intuitionistic fuzzy implication $\rightarrow_{189}$. Notes on Intuitionistic Fuzzy Sets, 23 (1), 14-20.

[11] Mendelson, E. (1964). Introduction to Mathematical Logic, Princeton, NJ: D. Van Nostrand.

[12] Vassilev, P., Ribagin, S., \& Kacprzyk, J. (2018) A remark on intuitionistic fuzzy implications. Notes on Intuitionistic Fuzzy Sets, 24 (2), 1-7. 